\title{
Synthesis and Antitumor Activity of Methoxy-indolo[2,1-a]isoquinolines
}

\author{
Reinhard Ambros, Silvia von Angerer*, and Wolfgang \\ Wiegrebe
}

Institut für Pharmazie, Lehrstuhl Pharmazeutische Chemie I and Sonderforschungsbereich 234, Universität Regensburg, Universitätsstr. 31, D-8400 Regensburg, Federal Republic of Germany

Received January 19, 1988

Methoxy-indolol2,1-alisoquinolines 8a-f and their dihydroderivatives 7a-f were synthesized by Bischler-Napieralski reaction of the (bromomethoxyphenyl)-[2-(methoxyphenyl)-ethyl]acetamides $4 \mathbf{a}-\mathbf{f}$, reduction, subsequent cyclization and dehydrogenation. They were tested for cytostatic activity in vitro using P388 $\mathrm{D}_{1}$ leukemia and MDA MB 231 mammary tumor cells. The trimethoxy-5,6-dihydroindoloisoquinoline 7d and the tetramethoxyindoloisoquinoline $\mathbf{8 f}$ showed an inhibition of cellproliferation of about $70 \%$ at a concentration of $10^{-5}$ molar.
Synthese und Antitumoraktivität von Methoxy-indolo[2,1-a]isochinolinen

Die Methoxy-indolo[2,1-a] isochinoline 8a-f und deren Dihydroderivate 7a-f wurden durch Bischler-Napieralski-Ringschluß der (Brom-methoxyphenyl)-[2-(methoxyphenyl)-ethyl]acetamide 4a-f, Reduktion, Cyclisierung und Dehydrierung gewonnen. Die cytostatische Wirkung wurde in vitro an der P388 $\mathrm{D}_{1}$ - und der MDA-MB 231-Zellinie getestet. Das Trimethoxy-5,6-dihydroindoloisochinolin $\mathbf{7 d}$ und das Tetramethoxyindoloisochinolin 8 feigten eine Hemmung der Zellproliferation von $70 \%$ bei einer Konzentration von $10^{-5} \mathrm{M}$.
The aim of our investigations is the synthesis of cytostatic compounds with binding affinity for the estrogen receptor, that can be used for the selective treatment of hormone dependent mammary tumors. Suitable structures for this approach are tetracyclic $\mathrm{N}$-heterocycles, which are known to intercalate into the $\mathrm{DNA}^{1)}$ and are able to bind to the estrogen receptor ${ }^{2}$.

Based on these findings, we synthesized a number of indolo[2,1-a]isoquinolines 8a-f and their dihydro analogues 7a-f. Cytostatic activity of these compounds was evaluated in vitro using MDA-MB 231 mammary tumor cells and P388 $\mathrm{D}_{1}$ leukemia cells.

\section{Chemistry}

The synthesis was performed as outlined in scheme 1 . The starting methyl bromophenylacetates $\mathbf{2 a - c}$ were synthesized by bromination of the corresponding phenylacetic acids and conversion to the methyl esters. The 2-phenylethylamine 3a was obtained directly by $\mathrm{LiAlH}_{4}$ reduction of the 3-methoxy- $\beta$-nitrostyrene ${ }^{3)}$ or, in better yield, in two steps with $\mathrm{NaBH}_{4}$ followed by $\mathrm{LiAlH}_{4}$. The best method was the hydrogenation of the 3-methoxy-phenylacetonitrile with $\mathrm{Rh} / \mathrm{C}$.

The reaction of the bromo-phenylacetates $\mathbf{2 a - c}$ with the 2 phenylethylamines $\mathbf{3 a}$ and $\mathbf{3} \mathbf{b}$ afforded the corresponding amides 4a-f. Cyclisation to the 3,4-dihydroisoquinolines was accomplished by a modified Bischler-Napieralski method using $\mathrm{POCl}_{3}$ in $\mathrm{CH}_{3} \mathrm{CN}$. The crude products were treated with $\mathrm{NaBH}_{4}$ to give the 1,2,3,4-tetrahydro-1-benzylisoquinolines 5a-f. In the case of $4 \mathbf{c}$ (2-Bromo-4,5-dimethoxyphenyl)-(3,4-dihydro-6-methoxyisoquinolyl-1)-ketone was formed as a byproduct, probably by air oxidation ${ }^{4}$. The correct substitution pattern in the isoquinoline ring was confirmed by ${ }^{1} \mathrm{H}-\mathrm{NMR}$ spectroscopy.

The benzylisoquinolines $\mathbf{5 a - f}$ were converted into the tetracyclic indolo[2,1-a]isoquinolines by treatment with $\mathrm{NaH}$ in DMSO. This reaction must involve a benzyne intermediate $^{5)}$ because both bromo compounds $\mathbf{5 a}$ and $\mathbf{5 b}$ led to the same structure. The reaction mixture contained two products
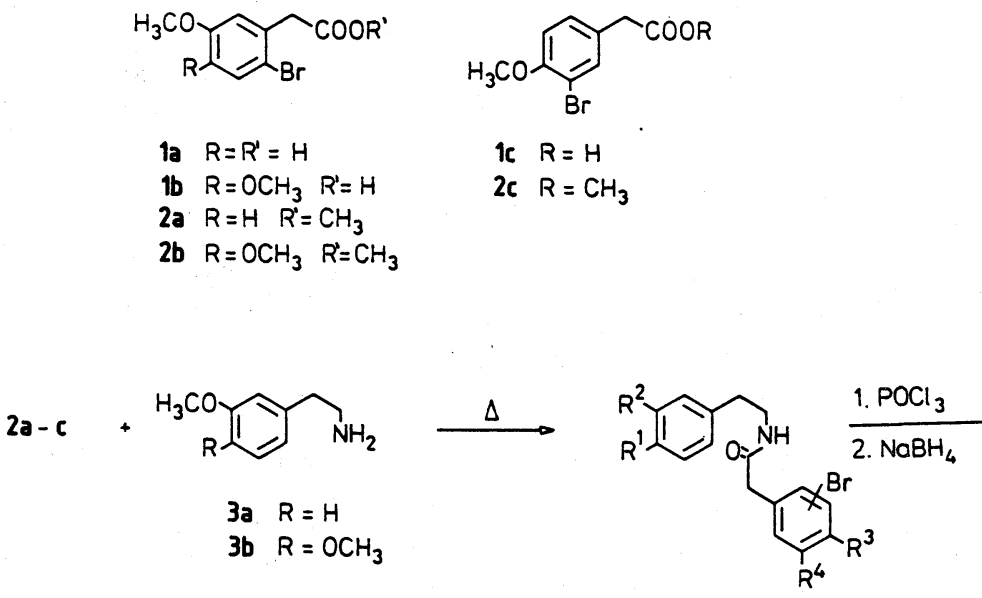

$4 a-f$

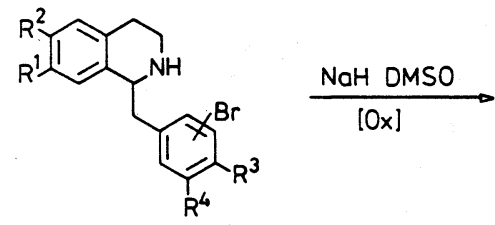

$5 a-f$

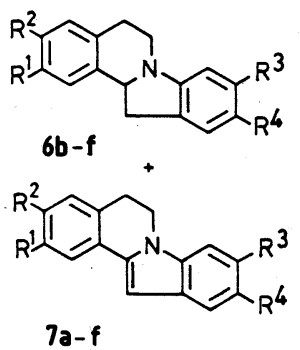

\begin{tabular}{|c|l|l|l|l|}
\hline $4-8$ & $R^{7}$ & $R^{2}$ & $R^{3}$ & $R^{4}$ \\
\hline a & $\mathrm{H}$ & $\mathrm{OCH}_{3}$ & $\mathrm{H}$ & $\mathrm{OCH}_{3}$ \\
b & $\mathrm{H}$ & $\mathrm{OCH}_{3}$ & $\mathrm{OCH}_{3}$ & $\mathrm{H}$ \\
c & $\mathrm{H}$ & $\mathrm{OCH}_{3}$ & $\mathrm{OCH}_{3}$ & $\mathrm{OCH}_{3}$ \\
d & $\mathrm{OCH}_{3}$ & $\mathrm{OCH}_{3}$ & $\mathrm{H}$ & $\mathrm{OCH}_{3}$ \\
$\mathrm{f}$ & $\mathrm{OCH}_{3}$ & $\mathrm{OCH}_{3}$ & $\mathrm{OCH}_{3}$ & $\mathrm{H}$ \\
\hline
\end{tabular}

$7 a-f$<smiles>[R]C1=CC2=Cc3cc([R])c([R3])cc3C3=C([R])C=C1C=CN23</smiles>

$8 a-f$ 
in a ratio of 1:4 which can be separated by column chromatography (CC). The expected tetrahydroindoloisoquinoline was only the minor product, whereas the main fraction contained the dihydro derivative. ${ }^{1} \mathrm{H}-\mathrm{NMR}$ spectroscopy revealed, that under the reaction conditions oxidation occures to the indoles 7a-f: the double dublett for the hydrogen at C-12a had disappeared and two tripletts for the hydrogen atoms at C-5 and C- 6 appeared instead of the complex multiplett in 6b-f.

This oxidation reaction can also be performed with DDQ or with $\mathrm{Pd} / \mathrm{C}$ at a temp. just above the melting point of the tetrahydro compound. Heating the dihydro derivative in the presence of $\mathrm{Pd} / \mathrm{C}$ above its melting point, which is considerably higher than that of the tetrahydro compound, afforded the aromatic indoloisoquinolines $\mathbf{8 a}-\mathbf{f}$.

\section{Cytostatic Activity}

Two different cell lines were used for the determination of cytostatic activity. The P388 $\mathrm{D}_{1}$ cell line derives from a mouse leukemia, the MDA-MB 231 cells are hormone-independent mammary tumor cells of human origin. All of the new indoloisoquinolines were tested for cytostatic effects at a concentration of $10^{-5}$ molar. The inhibition of cell growth was measured by cell counting and ${ }^{3} \mathrm{H}$-thymidine labeling. The tetrahydro derivatives $\mathbf{6 b}-\mathbf{f}$ were devoid of activity, probably due to their nonplanar structure. The dihydro compounds 7 showed a weak inhibitory effect except compound 7d that proved to be active against MDA-MB 231 (Table 1). Going to the aromatic indoloisoquinolines $\mathbf{8 a}-\mathbf{f}$, no significant increase of cytostatic activity was observed. The tetramethoxy derivative $\mathbf{8 f}$ was the most active compound in this series (Table 2).

These results showed, that cytostatic activity is not generally associated with a planar structure of the tetracyclic heterocycles. Compounds with a significant inhibition of cell growth possess a dimethoxyisoquinoline fragment (8d, 8f, 7d). This effect was not very marked, since a concentration of $10^{-5} \mathrm{M}$ was required for it. The rather low activity of this type of tetracycles may be due to the lack of a positive charge on the nitrogen.

The authors thank C. Braun and M. Beer for technical assistance and the Deutsche Forschungsgemeinschaft (SFB 234) for financial support.

\section{Experimental Part}

Melting points: Büchi 510 apparatus, uncorrected. - Elemental analyses: Mikroanalytisches Laboratorium, University of Regensburg. - IR-spectra: Beckman Acculab 3; KBr. $-{ }^{1} \mathrm{H}-\mathrm{NMR}$ spectra: Varian EM 360 A (60 $\mathrm{MHz}$ ); TMS as internal standard. - Temp. in ${ }^{\circ} \mathrm{C}$.

General Procedure for the Synthesis of the Bromo-phenylacetic acids 1a-c

Bromine $(17 \mathrm{ml}, 0.23 \mathrm{~mol})$ was added slowly to a solution of methoxyphenylacetic acid $(0.24 \mathrm{~mol})$ (Janssen) and $10.7 \mathrm{~g}(0.27 \mathrm{~mol})$ of $\mathrm{NaOH}$ in $400 \mathrm{ml}$ water at $50^{\circ}$. After stirring for $30 \mathrm{~min}$ and cooling to room temp., the precipitate was filtered off and washed with water. Recrystallization from aqueous $\mathrm{MeOH}$ yielded colorless crystals.

Tab. 1: Effect of 7a-f on the growth of MDA-MB 231 and P388 $D_{1}$ cells

\begin{tabular}{|c|c|c|c|c|}
\hline \multirow[b]{2}{*}{ Compound $^{\text {a) }}$} & \multicolumn{2}{|c|}{ P $388 D_{1}$} & \multicolumn{2}{|c|}{ MDA-MB 231} \\
\hline & $\begin{array}{l}\text { cell no. }{ }^{b)} \\
\% \mathrm{~T} / \mathrm{C}^{\mathrm{d})}\end{array}$ & $\begin{array}{c}{ }^{3} \mathrm{H} \text {-thymidine } \\
\text { incorp.c) } \\
\% \mathrm{~T} / \mathrm{C}^{\mathrm{d})}\end{array}$ & $\begin{array}{l}\text { cell no. }{ }^{b)} \\
\% \mathrm{~T} / \mathrm{C}^{\mathrm{d})}\end{array}$ & $\begin{array}{c}{ }^{3} \mathrm{H} \text {-thymidine } \\
\text { incorp.c) } \\
\% \mathrm{~T} / \mathrm{C}^{\mathrm{d})}\end{array}$ \\
\hline $7 a$ & 95 & 98 & 90 & 90 \\
\hline $7 \mathrm{~b}$ & 100 & 97 & 100 & 98 \\
\hline $7 c$ & 93 & 93 & 87 & 90 \\
\hline $7 d$ & 96 & 92 & 32 & 16 \\
\hline $\left.7 d^{e}\right)$ & & & 90 & 90 \\
\hline $7 e$ & 96 & 93 & 87 & 62 \\
\hline $7 f$ & 93 & 93 & 93 & 92 \\
\hline
\end{tabular}

a) Concentration $10^{-5} \mathrm{M}$.

b) Mean of three tests with six dishes/test tubes.

c) cpm $/ 10^{6}$ cells.

d) $\% \mathrm{~T} / \mathrm{C}=$ test compound/control $\times 100$.

e) Concentration $10^{-6} \mathrm{M}$.

Tab. 2: Effect of $8 \mathrm{a}-\mathrm{f}$ on the growth of MDA-MB 231 and P388 $\mathrm{D}_{1}$ cells

\begin{tabular}{|c|c|c|c|c|}
\hline \multirow[b]{2}{*}{ Compound ${ }^{a)}$} & \multicolumn{2}{|c|}{ P388 $D_{1}$} & \multicolumn{2}{|c|}{ MDA-MB 231} \\
\hline & $\begin{array}{l}\text { cell no. }^{\mathrm{b})} \\
\% \mathrm{~T} / \mathrm{C}^{\mathrm{d})}\end{array}$ & $\begin{array}{c}{ }^{3} \mathrm{H} \text {-thymidine } \\
\text { incorp.c) } \\
\% \mathrm{~T} / \mathrm{C}^{\mathrm{d})}\end{array}$ & $\begin{array}{l}\text { cell no. }{ }^{b)} \\
\left.\% \mathrm{~T} / \mathrm{C}^{\mathrm{d}}\right)\end{array}$ & $\begin{array}{c}{ }^{3} \text { H-thymidine } \\
\text { incorp.c) } \\
\% \mathrm{~T} / \mathrm{C}^{\mathrm{d})}\end{array}$ \\
\hline $\begin{array}{l}8 \mathrm{a} \\
8 \mathrm{~b} \\
\mathbf{8 c} \\
\mathbf{8 d} \\
\mathbf{8 e} \\
\mathbf{8 f}\end{array}$ & $\begin{array}{l}89 \\
78 \\
88 \\
71 \\
73 \\
30\end{array}$ & $\begin{array}{l}92 \\
84 \\
95 \\
73 \\
78 \\
15\end{array}$ & $\begin{array}{l}82 \\
80 \\
84 \\
48 \\
97 \\
48\end{array}$ & $\begin{array}{r}85 \\
85 \\
90 \\
45 \\
100 \\
21\end{array}$ \\
\hline
\end{tabular}

$a, b, c, d)$ see Tab. 1 . 


\section{2-Bromo-5-methoxy-phenylacetic acid (1a)}

Yield $90 \%$; m.p. $115^{\circ}$ (lit. 114-115 ${ }^{6}$ ) $)$. $-{ }^{1} \mathrm{H}-\mathrm{NMR}\left(\mathrm{CDCl}_{3}\right): \delta(\mathrm{ppm})=$ $3.8\left(\mathrm{~s} ; 5 \mathrm{H},-\mathrm{OCH}_{3},-\mathrm{CH}_{2}-\right), 6.67-6.9(\mathrm{~m} ; 2 \mathrm{H}, \mathrm{ArH}), 7.47(\mathrm{~d} ; \mathrm{J}=9 \mathrm{~Hz}, 1 \mathrm{H}$, ArH), 7.96 (s broad; $1 \mathrm{H}, \mathrm{OH})$.

\section{2-Bromo-4,5-dimethoxy-phenylacetic acid (1b)}

Yield $90 \%$; m.p. $115^{\circ}\left(\right.$ lit. $\left.115-116^{\circ}{ }^{6}\right)$ ). $-{ }^{1} \mathrm{H}-\mathrm{NMR}\left(\mathrm{CDCl}_{3}\right): \delta(\mathrm{ppm})=$ $3.77\left(\mathrm{~s} ; 2 \mathrm{H},-\mathrm{CH}_{2}-\right), 3.88\left(\mathrm{~s} ; 6 \mathrm{H},-\mathrm{OCH}_{3}\right), 6.76(\mathrm{~s} ; 1 \mathrm{H}, \mathrm{ArH}), 7.02(\mathrm{~s} ; 1 \mathrm{H}$, ArH), 8.16 (s broad; $1 \mathrm{H}, \mathrm{OH}$ ).

\section{3-Bromo-4-methoxy-phenylacetic acid (1c)}

Yield $80 \%$; m.p. $115^{\circ}$ (lit. $\left.115^{\circ} 7\right)$ ). ${ }^{1} \mathrm{H}-\mathrm{NMR}\left(\mathrm{CDCl}_{3}\right): \delta(\mathrm{ppm})=3.8$ (s; $\left.5 \mathrm{H},-\mathrm{OCH}_{3},-\mathrm{CH}_{2}-\right), 6.67-6.9(\mathrm{~m} ; 2 \mathrm{H}, \mathrm{ArH}), 7.47(\mathrm{~d} ; \mathrm{J}=9 \mathrm{~Hz}, 1 \mathrm{H}$, ArH), 7.96 (s broad; 1H, OH).

General Procedure for the Synthesis of the Methyl Bromo-methoxyphenylacetates $\mathbf{2 a - c}$

Bromo-methoxyphenylacetic acid $(0.23 \mathrm{~mol})$ in $180 \mathrm{ml}$ absol. $\mathrm{MeOH}$ and $6 \mathrm{ml}$ conc. $\mathrm{H}_{2} \mathrm{SO}_{4}$ was boiled for $17 \mathrm{~h}$. The volume of the solution was reduced to $50 \mathrm{ml}$. After addition of $100 \mathrm{ml}$ of water, the mixture was extracted with $\mathrm{CH}_{2} \mathrm{Cl}_{2}$. The org. layer was dried $\left(\mathrm{Na}_{2} \mathrm{SO}_{4}\right)$ and the solution was evaporated.

\section{Methyl 2-bromo-5-methoxyphenylacetate (2a)}

The product was purified by Kugelrohr-distillation: colorless oil. - Yield $90 \%$; b.p. $77-80^{\circ}, 0.2 \mathrm{~mm} .-\mathrm{C}_{10} \mathrm{H}_{11} \mathrm{BrO}_{3}$ (259.0) Calc. C $46.4 \mathrm{H} 4.25$ Found C $47.0 \mathrm{H} 4.61$. - IR (film): 1740 (CO) $\mathrm{cm}^{-1}$. - ${ }^{1} \mathrm{H}-\mathrm{NMR}\left(\mathrm{CDCl}_{3}\right)$ : $\delta(\mathrm{ppm})=3.70\left(\mathrm{~s} ; 2 \mathrm{H},-\mathrm{CH}_{2}-\right), 3.76\left(\mathrm{~s} ; 3 \mathrm{H}, \mathrm{COOCH}_{3}\right), 3.80(\mathrm{~s} ; 3 \mathrm{H}$, $\left.-\mathrm{OCH}_{3}\right), 6.6-6.9(\mathrm{~m} ; 2 \mathrm{H}, \mathrm{ArH}), 7.43(\mathrm{~d} ; \mathrm{J}=9 \mathrm{~Hz}, 1 \mathrm{H}, \mathrm{ArH})$.

\section{Methyl 2-bromo-4,5-dimethoxyphenylacetate (2b)}

The product was recrystallized from aqueous $\mathrm{MeOH}$ : colorless crystals. Yield $80 \%$; m.p. $48^{\circ}$. $-\mathrm{C}_{11} \mathrm{H}_{13} \mathrm{BrO}_{4}(289.1)$ Calc. C $45.7 \mathrm{H} 4.53$ Found C 45.4 H 4.68. - IR (KBr): $1740(\mathrm{CO}) \mathrm{cm}^{-1}$. - ${ }^{1} \mathrm{H}-\mathrm{NMR}\left(\mathrm{CDCl}_{3}\right)$ : $\delta(\mathrm{ppm})=3.77\left(\mathrm{~s} ; 5 \mathrm{H}, \mathrm{COOCH}_{3},-\mathrm{CH}_{2}-\right), 3.91\left(\mathrm{~s} ; 6 \mathrm{H},-\mathrm{OCH}_{3}\right), 6.87(\mathrm{~s}$; $1 \mathrm{H}, \mathrm{ArH}), 7.13$ (s; 1H, ArH).

\section{Methyl 3-bromo-4-methoxyphenylacetate (2c)}

Recrystallization from aqueous $\mathrm{MeOH}$ yielded colorless crystals. Yield $90 \%$; m.p. $31^{\circ}$ (lit. $48^{\circ}{ }^{8)}$ ). $-\mathrm{C}_{10} \mathrm{H}_{11} \mathrm{BrO}_{3}$ (259.0) Calc. C $46.4 \mathrm{H} 4.25$ Found C $46.2 \mathrm{H} \mathrm{4.35}$. - IR(KBr): $1740(\mathrm{CO}) \mathrm{cm}^{-1}$. - ${ }^{1} \mathrm{H}-\mathrm{NMR}\left(\mathrm{CDCl}_{3}\right)$ : $\delta(\mathrm{ppm})=3.6\left(\mathrm{~s} ; 2 \mathrm{H},-\mathrm{CH}_{2}-\right), 3.73\left(\mathrm{~s} ; 3 \mathrm{H},-\mathrm{COOCH}_{3}\right), 3.9(\mathrm{~s} ; 3 \mathrm{H}$, $\left.-\mathrm{OCH}_{3}\right), 6.86(\mathrm{~d} ; \mathrm{J}=9 \mathrm{~Hz}, 1 \mathrm{H}, \mathrm{ArH}), 7.23\left(\mathrm{dd} ; J_{1,2}=9 / 2 \mathrm{~Hz}, 1 \mathrm{H}\right.$, $\operatorname{ArH}), 7.5(\mathrm{~d} ; \mathrm{J}=2 \mathrm{~Hz}, 1 \mathrm{H}, \mathrm{ArH})$.

\section{1-Amino-2-(3-methoxyphenyl)-ethane (3a)}

$1 \mathrm{~g} \mathrm{Rh} / \mathrm{C} 10 \%$ was added to a solution of $50 \mathrm{~g}(0.34 \mathrm{~mol}) 3$-methoxy-phenylacetonitrile (Janssen) dissolved in $110 \mathrm{ml} \mathrm{EtOH}$ saturated with $\mathrm{NH}_{3}$. The mixture was hydrogenated at 30 bar for $7 \mathrm{~d}$. The catalyst was filtered off, washed with EtOH, and the solvent was evaporated. Pure 3a was obtained as colorless oil. Yield $98 \%$; b.p. $122-123^{\circ}, 7 \mathrm{~mm}$ (lit. 122-123, $\left.7 \mathrm{~mm}^{9}\right)$ ). ${ }^{1} \mathrm{H}-\mathrm{NMR}\left(\mathrm{CDCl}_{3}\right): \delta(\mathrm{ppm})=1.48\left(\mathrm{~s} ; 2 \mathrm{H},-\mathrm{NH}_{2}\right), 2.82(\mathrm{mc}$ $\left.4 \mathrm{H},-\mathrm{CH}_{2}-\right), 3.79\left(\mathrm{~s} ; 3 \mathrm{H},-\mathrm{OCH}_{3}\right), 6.59-7.3(\mathrm{~m} ; 4 \mathrm{H}, \mathrm{ArH})$.

\section{General Procedure for the Synthesis of the Acetamides 4a-f}

A flask containing $0.75 \mathrm{~mol}$ methoxy-phenylethylamine and $0.75 \mathrm{~mol} \mathrm{me}-$ thyl bromo-phenylacetate was placed into a hot oil bath. The temp. was kept at $150-155^{\circ}$ for $10 \mathrm{~h}$. After cooling to $35^{\circ} 10 \mathrm{ml}$ EtOAc were added with stirring. The product crystallizing at $4^{\circ}$ was filtered off and washed with ether. Acetamides, which did not crystallize spontaneously, were purified by $\mathrm{CC}\left(\mathrm{SiO}_{2}\right.$; ether/ $\left.\mathrm{CHCl}_{3} 1: 1\right)$ and crystallized from EtOAc/ether. The crystals of $\mathbf{4 a - f}$ are colorless.

\section{2-(2-Bromo-5-methoxyphenyl)-N-/2-(3-methoxyphenyl-ethyll-acetamide} (4a)

4a was synthesized from $2 \mathbf{a}$ and $3 \mathbf{a}$ and recrystallized from EtOH. Yield $50 \%$; m.p. 91 ${ }^{\circ}$. $-\mathrm{C}_{18} \mathrm{H}_{20} \mathrm{BrNO}_{3}$ (378.3) Calc. C $57.2 \mathrm{H} 5.35$ Found C 57.2 H 5.28. - IR(KBr): $3300(\mathrm{NH}), 1650,1555(\mathrm{CO}) \mathrm{cm}^{-1}$. $-{ }^{1} \mathrm{H}-$ $\operatorname{NMR}\left(\mathrm{CDCl}_{3}\right): \delta(\mathrm{ppm})=2.80\left(\mathrm{t} ; \mathrm{J}=7 \mathrm{~Hz}, 2 \mathrm{H},-\mathrm{CH}_{2}-\right), 3.53(\mathrm{t} ; \mathrm{J}=7 \mathrm{~Hz}$, $\left.2 \mathrm{H},-\mathrm{CH}_{2}-\right), 3.67\left(\mathrm{~s} ; 2 \mathrm{H},-\mathrm{COCH}_{2}-\right), 3.83\left(\mathrm{~s} ; 3 \mathrm{H},-\mathrm{OCH}_{3}\right), 3.93(\mathrm{~s} ; 3 \mathrm{H}$, $\left.-\mathrm{OCH}_{3}\right), 5.53$ (s broad; 1H, -NH), 6.67-7.43 (m; 6H, ArH), $7.77(\mathrm{~s} ; 1 \mathrm{H}$, ArH).

2-(3-Bromo-4-methoxyphenyl)-N-/2-(3-methoxyphenyl)-ethyll-acetamide (4b)

4b was synthesized from $\mathbf{2 c}$ and $\mathbf{3 a}$ and recrystallized from EtOAc/ether (7+3). Yield $55 \%$; m.p. 63-64 ${ }^{\circ}$. $-\mathrm{C}_{18} \mathrm{H}_{20} \mathrm{BrNO}_{3}$ (378.3) Calc. C 57.2 H 5.35 Found C $56.8 \mathrm{H} 5.43 .-{ }^{1} \mathrm{H}-\mathrm{NMR}\left(\mathrm{CDCl}_{3}\right): \delta(\mathrm{ppm})=2.73(\mathrm{t} ; \mathrm{J}=$ $\left.7 \mathrm{~Hz}, 2 \mathrm{H},-\mathrm{CH}_{2}-\right), 3.3-3.53\left(\mathrm{~m} ; 4 \mathrm{H},-\mathrm{COCH}_{2}-,-\mathrm{CH}_{2}-\right), 3.77(\mathrm{~s} ; 3 \mathrm{H}$, $\left.-\mathrm{OCH}_{3}\right), 3.87\left(\mathrm{~s} ; 3 \mathrm{H},-\mathrm{OCH}_{3}\right), 5.49$ (s broad; $\left.1 \mathrm{H},-\mathrm{NH}\right), 6.56-6.79(\mathrm{~m}$; $3 \mathrm{H}, \mathrm{ArH}), 6.86(\mathrm{~s} ; 1 \mathrm{H}, \mathrm{ArH}), 7.0-7.17$ (m; 2H, ArH), $7.34(\mathrm{~d} ; \mathrm{J}=2 \mathrm{~Hz}$, $1 \mathrm{H}, \mathrm{ArH})$.

\section{2-(2-Bromo-4,5-dimethoxyphenyl)-N-/2-(3-methoxyphenyl)-ethyll-acet- amide (4c)}

$\mathbf{4 c}$ was synthesized from $\mathbf{2 b}$ and $\mathbf{3 a}$ and recrystallized from EtOH. Yield $60 \%$; m.p. $145-146.5^{\circ}$. $-\mathrm{C}_{19} \mathrm{H}_{22} \mathrm{BrNO}_{4}$ (408.3) Calc. C $55.9 \mathrm{H} 5.39$ Found C $55.8 \mathrm{H} \mathrm{5.40.} \mathrm{-}{ }^{1} \mathrm{H}-\mathrm{NMR}\left(\mathrm{CDCl}_{3}\right): \delta(\mathrm{ppm})=2.77(\mathrm{t} ; \mathrm{J}=7 \mathrm{~Hz}$, $\left.2 \mathrm{H},-\mathrm{CH}_{2}-\right), 3.47\left(\mathrm{t} ; \mathrm{J}=7 \mathrm{~Hz}, 2 \mathrm{H},-\mathrm{CH}_{2}-\right), 3.63\left(\mathrm{~s} ; 2 \mathrm{H},-\mathrm{COCH}_{2}-\right), 3.82(\mathrm{~s}$; $\left.3 \mathrm{H},-\mathrm{OCH}_{3}\right), 3.85\left(\mathrm{~s} ; 3 \mathrm{H},-\mathrm{OCH}_{3}\right), 3.90\left(\mathrm{~s} ; 3 \mathrm{H},-\mathrm{OCH}_{3}\right), 5.50$ (s broad; $1 \mathrm{H},-\mathrm{NH}), 6.65-7.33(\mathrm{~m} ; 6 \mathrm{H}, \mathrm{ArH})$.

\section{2-(2-Bromo-5-methoxyphenyl)-N-/2-(3,4-dimethoxyphenyl)-ethyll-acet- amide (4d)}

4d was synthesized from 2a and 1-amino-2-(3,4-dimethoxyphenyl)-ethane (3b) (Janssen) and recrystallized from EtOH. Yield $55 \%$; m.p. 128-129 . $-\mathrm{C}_{19} \mathrm{H}_{22} \mathrm{BrNO}_{4}(408.3)$ Calc. C $55.9 \mathrm{H} 5.39$ Found C $55.4 \mathrm{H} 5.70 .{ }^{1} \mathrm{H}-$ $\mathrm{NMR}\left(\mathrm{CDCl}_{3}\right): \delta(\mathrm{ppm})=2.70\left(\mathrm{t} ; \mathrm{J}=7 \mathrm{~Hz}, 2 \mathrm{H},-\mathrm{CH}_{2}-\right) 3.42(\mathrm{t} ; \mathrm{J}=7 \mathrm{~Hz}$, $\left.2 \mathrm{H},-\mathrm{CH}_{2}-\right), 3.60\left(\mathrm{~s} ; 2 \mathrm{H},-\mathrm{COCH}_{2}-\right), 3.73\left(\mathrm{~s} ; 3 \mathrm{H},-\mathrm{OCH}_{3}\right), 3.80(\mathrm{~s} ; 3 \mathrm{H}$, $\left.-\mathrm{OCH}_{3}\right), 3.82\left(\mathrm{~s} ; 3 \mathrm{H},-\mathrm{OCH}_{3}\right), 5.53$ (s broad; $\left.1 \mathrm{H},-\mathrm{NH}\right), 6.63-6.85(\mathrm{~m}$; $5 \mathrm{H}, \mathrm{ArH}), 7.43(\mathrm{~d} ; \mathrm{J}=9 \mathrm{~Hz}, 1 \mathrm{H}, \mathrm{ArH})$.

2-(3-Bromo-4-methoxyphenyl)-N-/2,-(3,4-dimethoxyphenyl)-ethyll-acetamide (4e)

$4 \mathbf{e}$ was synthesized from $2 \mathbf{c}$ and $3 \mathbf{b}$ and recrystallized from EtOH. Yield $50 \%$; m.p. $123-124^{\circ}$. $-\mathrm{C}_{19} \mathrm{H}_{22} \mathrm{BrNO}_{4}$ (408.3) Calc. C $55.9 \mathrm{H} 5.39$ Found C $55.9 \mathrm{H} \mathrm{5.44.}-{ }^{1} \mathrm{H}-\mathrm{NMR}\left(\mathrm{CDCl}_{3}\right): \delta(\mathrm{ppm})=2.73(\mathrm{t} ; \mathrm{J}=7 \mathrm{~Hz}$, $\left.2 \mathrm{H},-\mathrm{CH}_{2}-\right), 3.42\left(\mathrm{t} ; \mathrm{J}=7 \mathrm{~Hz}, 2 \mathrm{H},-\mathrm{CH}_{2}-\right), 3.47\left(\mathrm{~s} ; 2 \mathrm{H},-\mathrm{COCH}_{2}-\right), 3.88(\mathrm{~s}$; $\left.3 \mathrm{H},-\mathrm{OCH}_{3}\right), 3.93\left(\mathrm{~s} ; 3 \mathrm{H},-\mathrm{OCH}_{3}\right), 3.95\left(\mathrm{~s} ; 3 \mathrm{H},-\mathrm{OCH}_{3}\right), 5.55$ (s broad; $1 \mathrm{H},-\mathrm{NH}), 6.67-7.0(\mathrm{~m} ; 4 \mathrm{H}, \mathrm{ArH}), 7.18\left(\mathrm{dd} ; \mathrm{J}_{1 / 2}=9 / 2 \mathrm{~Hz}, 1 \mathrm{H}, \mathrm{ArH}\right)$, $7.45(\mathrm{~d} ; \mathrm{J}=2 \mathrm{~Hz}, 1 \mathrm{H}, \mathrm{ArH})$.

\section{2-(2-Bromo-4,5-dimethoxyphenyl)-N-/2-(3,4-dimethoxyphenyl)-ethyll-} acetamide (4f)

$\mathbf{4 f}$ was synthesized from $\mathbf{2 b}$ and $\mathbf{3 b}$ and recrystallized from EtOH. Yield $60 \%$; m.p. $158.5-159^{\circ}$. $-\mathrm{C}_{20} \mathrm{H}_{24} \mathrm{BrNO}_{5}$ (438.3) Calc. C $54.8 \mathrm{H} 5.52$ Found C $54.9 \mathrm{H} \mathrm{5.53.}{ }^{1} \mathrm{H}-\mathrm{NMR}\left(\mathrm{CDCl}_{3}\right): \delta(\mathrm{ppm})=2.73(\mathrm{t} ; \mathrm{J}=7 \mathrm{~Hz}$, $\left.2 \mathrm{H},-\mathrm{CH}_{2}-\right), 3.47\left(\mathrm{t} ; \mathrm{J}=7 \mathrm{~Hz}, 2 \mathrm{H},-\mathrm{CH}_{2}-\right), 3.63\left(\mathrm{~s} ; 2 \mathrm{H},-\mathrm{COCH}_{2}-\right), 3.87$ (s; $12 \mathrm{H},-\mathrm{OCH}_{3}$ ), 5.57 (s broad; $\left.1 \mathrm{H},-\mathrm{NH}\right), 6.67-7.07$ (m; $\left.5 \mathrm{H}, \mathrm{ArH}\right)$. 
General Procedure for the Synthesis of the 1-Benzyl-1,2,3,4-tetrahydroisoquinolines $\mathbf{5 a - f}$

A mixture of $55 \mathrm{mmol}$ of acetamide, $20 \mathrm{ml}$ of $\mathrm{POCl}_{3}$ and $75 \mathrm{ml}$ of absol. $\mathrm{CH}_{3} \mathrm{CN}$ was refluxed for $4 \mathrm{~h}$. With cooling, $150 \mathrm{ml}$ of $20 \% \mathrm{NaOH}$ solution were added, the mixture was poured onto ice water and extracted with $\mathrm{CHCl}_{3}$. The $\mathrm{CHCl}_{3}$ solution was extracted with $150 \mathrm{ml} 2 \mathrm{~N} \mathrm{HCl}$. The free base was liberated with $20 \% \mathrm{NaOH}$ and extracted with $\mathrm{CHCl}_{3}$. The org. layer was washed with water and saline, and dried $\left(\mathrm{Na}_{2} \mathrm{SO}_{4}\right)$. The 3,4-dihydroisoquinolines obtained after evaporation of the solvent were used without further purification. The yields were between 55 and $70 \%$. Air must be excluded as far as possible during workup.

$\mathrm{NaBH}_{4}(0.11 \mathrm{~mol})$ was added slowly to a solution of $13.4 \mathrm{mmol}$ of 3,4-dihydroisoquinoline in $100 \mathrm{ml} \mathrm{MeOH}$ and $15 \mathrm{ml}$ water at $0^{\circ}$. The mixture was stirred for $2 \mathrm{~h}$ at room temp. After the solvent had been removed, the residue was treated with $100 \mathrm{ml}$ water and extracted with $\mathrm{CHCl}_{3}$. The org. layer was washed with water, and dried $\left(\mathrm{Na}_{2} \mathrm{SO}_{4}\right)$. After evaporation of the solvent the residue crystallized with aqueous $\mathrm{EtOH}$ or was purified by $\mathrm{CC}\left(\mathrm{SiO}_{2} ; \mathrm{CHCl}_{3}\right.$ /ether 1:1). Recrystallization from aqueous EtOH afforded colorless crystals. The yields were $58-80 \%$.

1-(2-Bromo-5-methoxybenzyl)-1,2,3,4-tetrahydro-6-methoxyisoquinoline (5a)

Yield $50 \%$; m.p. $72-74^{\circ}$. $-\mathrm{C}_{18} \mathrm{H}_{20} \mathrm{BrNO}_{2}$ (362.3) Calc. C $59.7 \mathrm{H} 5.56$ Found C $59.3 \mathrm{H} \mathrm{5.54.} \mathrm{-} \mathrm{IR} \mathrm{(KBr):} 3350(\mathrm{NH}) \mathrm{cm}^{-1}$. - ${ }^{1} \mathrm{H}-\mathrm{NMR}\left(\mathrm{CDCl}_{3}\right)$ : $\delta(\mathrm{ppm})=1.79(\mathrm{~s}$ broad; $1 \mathrm{H},-\mathrm{NH}), 2.67-3.53\left(\mathrm{~m} ; 6 \mathrm{H},-\mathrm{CH}_{2}-\right), 3.80(\mathrm{~s}$; $\left.6 \mathrm{H},-\mathrm{OCH}_{3}\right), 4.31\left(\mathrm{dd} ; \mathrm{J}_{1 / 2}=10 / 4 \mathrm{~Hz}, 1 \mathrm{H},-\mathrm{CH}-\mathrm{N}\right), 6.58-6.87(\mathrm{~m} ; 4 \mathrm{H}$, ArH), $7.26(\mathrm{~d} ; \mathrm{J}=9 \mathrm{~Hz}, 1 \mathrm{H}, \operatorname{ArH}), 7.48(\mathrm{~d} ; \mathrm{J}=9 \mathrm{~Hz}, 1 \mathrm{H}, \operatorname{ArH})$.

1-(3-Bromo-4-methoxybenzyl)-1,2,3,4-tetrahydro-6-methoxyisoquinoline (5b)

Yield $70 \%$; m.p. $97-98^{\circ}$. $-\mathrm{C}_{18} \mathrm{H}_{20} \mathrm{BrNO}_{2}$ (362.3) Calc. C $59.7 \mathrm{H} 5.56$ Found C $59.0 \mathrm{H} \mathrm{5.68.}-{ }^{1} \mathrm{H}-\mathrm{NMR}\left(\mathrm{CDCl}_{3}\right): \delta(\mathrm{ppm})=2.57(\mathrm{~s}$, broad; $1 \mathrm{H}$, $-\mathrm{NH}), 2.73-3.30\left(\mathrm{~m} ; 6 \mathrm{H},-\mathrm{CH}_{2}-\right), 3.77\left(\mathrm{~s} ; 3 \mathrm{H},-\mathrm{OCH}_{3}\right), 3.87(\mathrm{~s} ; 3 \mathrm{H}$, $\left.-\mathrm{OCH}_{3}\right), 4.15\left(\mathrm{dd} ; \mathrm{J}_{1 / 2}=10 / 4 \mathrm{~Hz}, 1 \mathrm{H},-\mathrm{CH}-\mathrm{N}\right), 6.59-6.82(\mathrm{~m} ; 3 \mathrm{H}, \mathrm{ArH})$, $6.88(\mathrm{~s} ; 1 \mathrm{H}, \mathrm{ArH}), 7.1-7.28(\mathrm{~m} ; 2 \mathrm{H}, \mathrm{ArH}), 7.47(\mathrm{~d} ; \mathrm{J}=2 \mathrm{~Hz}, 1 \mathrm{H}, \mathrm{ArH})$.

1-(2-Bromo-4,5-dimethoxybenzyl)-1,2,3,4-tetrahydro-6-methoxyisoquinoline (5c)

Yield $60 \%$; m.p. $114^{\circ}$. $-\mathrm{C}_{19} \mathrm{H}_{22} \mathrm{BrNO}_{3}$ (392.3) Calc. C 58.2 H 5.65 Found C 57.7 H 5.51. - ${ }^{1} \mathrm{H}-\mathrm{NMR}\left(\mathrm{CDCl}_{3}\right): \delta(\mathrm{ppm})=2.0(\mathrm{~s}$ broad; $1 \mathrm{H}$, $-\mathrm{NH}), 2.70-3.40\left(\mathrm{~m} ; 6 \mathrm{H},-\mathrm{CH}_{2}-\right), 3.83\left(\mathrm{~s} ; 3 \mathrm{H},-\mathrm{OCH}_{3}\right), 3.87(\mathrm{~s} ; 3 \mathrm{H}$, $\left.-\mathrm{OCH}_{3}\right), 3.92\left(\mathrm{~s} ; 3 \mathrm{H},-\mathrm{OCH}_{3}\right), 4.33\left(\mathrm{dd} ; \mathrm{J}_{1 / 2}=10 / 4 \mathrm{~Hz}, 1 \mathrm{H},-\mathrm{CH}-\mathrm{N}\right)$, 6.70-6.90 (m;3H, ArH), 7.15 (s; 1 H, ArH), $7.30(\mathrm{~d} ; \mathrm{J}=9 \mathrm{~Hz}, 1 \mathrm{H}, \mathrm{ArH})$.

\section{(2-Bromo-4,5-dimethoxyphenyl)-(3,4-dihydro-6-methoxyisoquinolyl-1-)-} ketone

This compound was formed as a byproduct of the cyclization of $4 \mathbf{c}$. It was purified by $\mathrm{CC}\left(\mathrm{SiO}_{2} ; \mathrm{CH}_{2} \mathrm{Cl}_{2}\right)$ to afford colorless crystals. Yield $20 \%$; m.p. $180-181^{\circ}$. $-\mathrm{C}_{19} \mathrm{H}_{18} \mathrm{BrNO}_{4}$ (404.3) Calc. C $56.4 \mathrm{H} 4.46$ Found C $56.0 \mathrm{H}$ 4.62. - IR(KBr): $1670(\mathrm{CO}) \mathrm{cm}^{-1}$. $-{ }^{1} \mathrm{H}-\mathrm{NMR}\left(\mathrm{CDCl}_{3}\right): \delta(\mathrm{ppm})$ $=2.80\left(\mathrm{t}: \mathrm{J}=7 \mathrm{~Hz}, 2 \mathrm{H},-\mathrm{CH}_{2}-\right), 3.80\left(\mathrm{t} ; \mathrm{J}=7 \mathrm{~Hz}, 2 \mathrm{H},-\mathrm{CH}_{2}-\right), 3.90(\mathrm{~s} ; 3 \mathrm{H}$, $\left.-\mathrm{OCH}_{3}\right), 3.95\left(\mathrm{~s} ; 6 \mathrm{H},-\mathrm{OCH}_{3}\right), 6.80-6.97(\mathrm{~m} ; 2 \mathrm{H}, \mathrm{ArH}), 7.27(\mathrm{~s} ; 1 \mathrm{H}$, $\operatorname{ArH}), 7.63(\mathrm{~d} ; \mathrm{J}=9 \mathrm{~Hz}, 1 \mathrm{H}, \operatorname{ArH})$.

1-(2-Bromo-5-methoxybenzyl)-1,2,3,4-tetrahydro-6,7-dimethoxyisoquinoline (5d)

Yield $65 \%$; m.p. $117^{\circ}$. - $\mathrm{C}_{19} \mathrm{H}_{22} \mathrm{BrNO}_{3}$ (392.3) Calc. C $58.2 \mathrm{H} 5.65$ Found C $58.0 \mathrm{H} \mathrm{5.55.}-{ }^{1} \mathrm{H}-\mathrm{NMR}\left(\mathrm{CDCl}_{3}\right): \delta(\mathrm{ppm})=1.90(\mathrm{~s}$ broad; $1 \mathrm{H}$, $-\mathrm{NH}), 2.67-3.50\left(\mathrm{~m} ; 6 \mathrm{H},-\mathrm{CH}_{2}-\right), 3.73\left(\mathrm{~s} ; 3 \mathrm{H},-\mathrm{OCH}_{3}\right), 3.77(\mathrm{~s} ; 3 \mathrm{H}$, $\left.-\mathrm{OCH}_{3}\right), 3.82\left(\mathrm{~s} ; 3 \mathrm{H},-\mathrm{OCH}_{3}\right), 4.33$ (dd; $\left.\mathrm{J}_{1 / 2}=10 / 4 \mathrm{~Hz}, 1 \mathrm{H},-\mathrm{CH}-\mathrm{N}\right), 6.57$ (s; $1 \mathrm{H}, \operatorname{ArH}), 6.58-6.83(\mathrm{~m} ; 2 \mathrm{H}, \operatorname{ArH}), 6.72(\mathrm{~s} ; 1 \mathrm{H}, \operatorname{ArH}), 7.47$ (d; J = $9 \mathrm{~Hz}, 1 \mathrm{H}, \mathrm{ArH})$.
1-(3-Bromo-4-methoxybenzyl)-1,2,3,4-tetrahydro-6,7-dimethoxyisoquinoline (5e)

Yield $55 \%$; m.p. $107-109^{\circ} .-\mathrm{C}_{19} \mathrm{H}_{22} \mathrm{BrNO}_{3}$ (392.3) Calc. C $58.2 \mathrm{H} 5.65$ Found C $57.8 \mathrm{H} 5.62 .{ }^{1} \mathrm{H}-\mathrm{NMR}\left(\mathrm{CDCl}_{3}\right): \delta(\mathrm{ppm})=1.80(\mathrm{~s}$ broad; $1 \mathrm{H}$, $-\mathrm{NH}), 2.67-3.50\left(\mathrm{~m} ; 6 \mathrm{H},-\mathrm{CH}_{2}^{-}\right), 3.88\left(\mathrm{~s} ; 3 \mathrm{H},-\mathrm{OCH}_{3}\right), 3.92(\mathrm{~s} ; 3 \mathrm{H}$, $\left.-\mathrm{OCH}_{3}\right), 3.95\left(\mathrm{~s} ; 3 \mathrm{H},-\mathrm{OCH}_{3}\right), 4.17\left(\mathrm{dd} ; \mathrm{J}_{1 / 2}=10 / 4 \mathrm{~Hz}, 1 \mathrm{H},-\mathrm{CH}-\mathrm{N}\right), 6.70$ (s; $2 \mathrm{H}, \operatorname{ArH}), 6.90$ (d; J $=9 \mathrm{~Hz}, 1 \mathrm{H}, \operatorname{ArH}), 7.27\left(\mathrm{dd} ; \mathrm{J}_{1 / 2}=9 / 2 \mathrm{~Hz}, 1 \mathrm{H}\right.$, $\operatorname{ArH}), 7.57(\mathrm{~d} ; \mathrm{J}=2 \mathrm{~Hz}, 1 \mathrm{H}, \operatorname{ArH})$.

1-(2-Bromo-4,5-dimethoxybenzyl)-1,2,3,4-tetrahydro-6,7-dimethoxyisoquinoline (5f)

Yield $80 \%$; m.p. $107-109^{\circ}\left(\right.$ lit. $\left.111^{\circ}{ }^{10}\right)$ ).

General Procedure for the Ring Closure of the Bromo-tetrahydrobenzyisoquinolines to the Tetrahydro- and Dihydro-indolo/ 2,1-alisoquinolines 6b-f and 7a-f

A solution of bromo-tetrahydro-benzylisoquinoline $(10 \mathrm{mmol})$ in $40 \mathrm{ml}$ DMSO was added to a solution of sodium methylsulfinylmethanide (prepared from $2.1 \mathrm{~g}(70 \mathrm{mmol}) \mathrm{NaH}(80 \%$ in oil dispersion) and $40 \mathrm{ml}$ DMSO). After stirring had been continued for $15 \mathrm{~h}$, the mixture was poured into $400 \mathrm{ml}$ water containing excess $\mathrm{NH}_{4} \mathrm{Cl}$ and extracted with $\mathrm{CHCl}_{3}$. The org. layer was washed with water and saline. After drying $\left(\mathrm{Na}_{2} \mathrm{SO}_{4}\right)$, evaporation of the solvent afforded a brownish oil, which was chromatographed $\left(\mathrm{SiO}_{2} ; \mathrm{CH}_{2} \mathrm{Cl}_{2}\right)$. The first fraction ( $\left.\mathrm{Rf} 0.7\right)$ contained the dihydroindoloisoquinolines as main product. The tetrahydro-indoloisoquinolines were isolated as second fraction ( $\mathrm{Rf} 0.3$ ). Both products were recrystallized from $\mathrm{EtOH}$ to afford colorless crystals. Their yields ranged from 40 to $70 \%$. - In the case of $\mathbf{5 a}$ no tetrahydro product was isolated.

\section{5,6,12,12a-Tetrahydro-3,9-dimethoxy-indolo[2,1-alisoquinoline (6b)}

M.p. 53-54 ${ }^{\circ}$. $-\mathrm{C}_{18} \mathrm{H}_{19} \mathrm{NO}_{2}(281.3)$ Calc. C $76.8 \mathrm{H} 6.81$ Found C 76.6 H 6.77. $-{ }^{1} \mathrm{H}-\mathrm{NMR}\left(\mathrm{CDCl}_{3}\right): \delta(\mathrm{ppm})=2.53-3.66\left(\mathrm{~m} ; 6 \mathrm{H},-\mathrm{CH}_{2}-\right), 3.76$ (s; $\left.3 \mathrm{H},-\mathrm{OCH}_{3}\right), 3.79\left(\mathrm{~s} ; 3 \mathrm{H},-\mathrm{OCH}_{3}\right), 4.69\left(\mathrm{dd} ; \mathrm{J}_{1 / 2}=8 / 3 \mathrm{~Hz}, 1 \mathrm{H}\right.$, $-\mathrm{CH}-\mathrm{N}), 6.1-7.2(\mathrm{~m} ; 6 \mathrm{H}, \mathrm{ArH})$.

\section{5,6,12,12a-Tetrahydro-3,9,10-trimethoxy-indolo/2,1-a/isoquinoline (6c)}

M.p. $101^{\circ}$. $-\mathrm{C}_{19} \mathrm{H}_{21} \mathrm{NO}_{3}(311.4)$ Calc. C $73.3 \mathrm{H} 6.80$ Found $\mathrm{C} 73.0$ $\mathrm{H}$ 6.89. - ${ }^{1} \mathrm{H}-\mathrm{NMR}\left(\mathrm{CDCl}_{3}\right): \delta(\mathrm{ppm})=2.43-3.67\left(\mathrm{~m} ; 6 \mathrm{H},-\mathrm{CH}_{2}-\right), 3.82$ (s; $\left.6 \mathrm{H},-\mathrm{OCH}_{3}\right), 3.93\left(\mathrm{~s} ; 3 \mathrm{H},-\mathrm{OCH}_{3}\right), 4.92\left(\mathrm{dd} ; \mathrm{J}_{1 / 2}=8 / 3 \mathrm{~Hz}, 1 \mathrm{H}\right.$, -CH-N), $6.40(\mathrm{~s} ; 1 \mathrm{H}, \mathrm{ArH}), 6.65-7.0(\mathrm{~m} ; 3 \mathrm{H}, \mathrm{ArH}), 7.27(\mathrm{~d} ; \mathrm{J}=9 \mathrm{~Hz}$, $1 \mathrm{H}, \mathrm{ArH})$.

\section{5,6,12,12a-Tetrahydro-2,3,10-trimethoxy-indolo/ 2,1-a/isoquinoline (6d)}

M.p. $128-129^{\circ} .-\mathrm{C}_{19} \mathrm{H}_{21} \mathrm{NO}_{3}(311.4)$ Calc. C $73.3 \mathrm{H} 6.80$ Found $\mathrm{C} 73.2$ H 6.80. - ${ }^{1} \mathrm{H}-\mathrm{NMR}\left(\mathrm{CDCl}_{3}\right): \delta(\mathrm{ppm})=2.33-3.80\left(\mathrm{~m} ; 6 \mathrm{H},-\mathrm{CH}_{2}-\right), 3.67$ (s; $\left.3 \mathrm{H},-\mathrm{OCH}_{3}\right), 3.77\left(\mathrm{~s} ; 3 \mathrm{H},-\mathrm{OCH}_{3}\right), 3.83\left(\mathrm{~s} ; 3 \mathrm{H},-\mathrm{OCH}_{3}\right), 4.82$ (dd; $\left.\mathrm{J}_{1 / 2}=8 / 3 \mathrm{~Hz}, 1 \mathrm{H},-\mathrm{CH}-\mathrm{N}\right), 6.45(\mathrm{~s} ; 1 \mathrm{H}, \mathrm{ArH}), 6.55-6.70(\mathrm{~m} ; 4 \mathrm{H}, \mathrm{ArH})$.

\section{5,6,12,12a-Tetrahydro-2,3,9-trimethoxy-indolo/ 2,Ialisoquinoline (6e)}

M.p. $120-121^{\circ}$. $-\mathrm{C}_{19} \mathrm{H}_{21} \mathrm{NO}_{3}$ (311.4). - Calc. C $73.3 \mathrm{H} 6.80$ Found C 73.2 H 6.79. $-{ }^{1} \mathrm{H}-\mathrm{NMR}\left(\mathrm{CDCl}_{3}\right): \delta(\mathrm{ppm})=2.50-3.77(\mathrm{~m} ; 6 \mathrm{H}$, $\left.-\mathrm{CH}_{2}-\right), 3.77\left(\mathrm{~s} ; 3 \mathrm{H},-\mathrm{OCH}_{3}\right), 3.83\left(\mathrm{~s} ; 3 \mathrm{H},-\mathrm{OCH}_{3}\right), 3.90\left(\mathrm{~s} ; 3 \mathrm{H},-\mathrm{OCH}_{3}\right)$, $4.87\left(\mathrm{dd} ; \mathrm{J}_{1 / 2}=8 / 3 \mathrm{~Hz}, 1 \mathrm{H},-\mathrm{CH}-\mathrm{N}\right), 6.10-6.27(\mathrm{~m} ; 2 \mathrm{H}, \mathrm{ArH}), 6.50$ (s; $1 \mathrm{H}, \operatorname{ArH}), 6.67(\mathrm{~s} ; 1 \mathrm{H}, \mathrm{ArH}), 6.95(\mathrm{~d} ; \mathrm{J}=9 \mathrm{~Hz}, 1 \mathrm{H}, \operatorname{ArH})$.

\section{5,6,12,12a-Tetrahydro-2,3,9,10-tetramethoxy-indolo/2,1 a/isoquinoline} (6f)

M.p. $105-107^{\circ}$ (lit. $\left.105-107^{\circ}{ }^{11)}\right)$. 


\section{5,6-Dihydro-3,10-dimethoxy-indolo/2,1-alisoquinoline (7a)}

M.p. $208^{\circ}$. $-\mathrm{C}_{18} \mathrm{H}_{17} \mathrm{NO}_{2}$ (279.3) Calc. C $77.4 \mathrm{H} 6.13$ Found C 76.9 $\mathrm{H}$ 6.05. $-{ }^{1} \mathrm{H}-\mathrm{NMR}\left(\mathrm{CDCl}_{3}\right): \delta(\mathrm{ppm})=3.16\left(\mathrm{t} ; \mathrm{J}=7 \mathrm{~Hz}, 2 \mathrm{H},-\mathrm{CH}_{2}-\right)$, $3.83\left(\mathrm{~s} ; 6 \mathrm{H},-\mathrm{OCH}_{3}\right), 4.19\left(\mathrm{t} ; \mathrm{J}=7 \mathrm{~Hz}, 2 \mathrm{H},-\mathrm{CH}_{2}-\right), 6.65-7.10(\mathrm{~m} ; 6 \mathrm{H}$, ArH, vinyl-H), 7.61 (d; J $=9 \mathrm{~Hz}, 1 \mathrm{H}, \mathrm{ArH})$.

\section{5,6-Dihydro-3,9-dimethoxy-indolo/2,1-a/isoquinoline (7b)}

M.p. $176^{\circ}$. $-\mathrm{C}_{18} \mathrm{H}_{17} \mathrm{NO}_{2}$ (279.3) Calc. C $77.4 \mathrm{H} 6.13$ Found $\mathrm{C} 77.6$ $\mathrm{H}$ 6.30. $-{ }^{1} \mathrm{H}-\mathrm{NMR}\left(\mathrm{CDCl}_{3}\right): \delta(\mathrm{ppm})=3.1\left(\mathrm{t} ; \mathrm{J}=7 \mathrm{~Hz}, 2 \mathrm{H},-\mathrm{CH}_{2}-\right), 3.77$ $\left(\mathrm{s} ; 3 \mathrm{H},-\mathrm{OCH}_{3}\right), 3.85\left(\mathrm{~s} ; 3 \mathrm{H},-\mathrm{OCH}_{3}\right), 4.13\left(\mathrm{t} ; \mathrm{J}=7 \mathrm{~Hz}, 2 \mathrm{H},-\mathrm{CH}_{2}-\right)$, 6.48-6.86 (m;5H, ArH, vinyl-H), $7.48(\mathrm{~d} ; \mathrm{J}=9 \mathrm{~Hz}, 1 \mathrm{H}, \mathrm{ArH}), 7.66(\mathrm{~d}$; $\mathrm{J}=9 \mathrm{~Hz}, 1 \mathrm{H}, \operatorname{ArH})$.

\section{5,6-Dihydro-3,9,10-trimethoxy-indolo/2,1-a/isoquinoline (7c)}

M.p. $212^{\circ}$. $-\mathrm{C}_{19} \mathrm{H}_{19} \mathrm{NO}_{3}(309.4)$ Calc. C 73.8 H 6.19 Found C 73.9 H 6.15. - ${ }^{1} \mathrm{H}-\mathrm{NMR}\left(\mathrm{CDCl}_{3}\right): \delta(\mathrm{ppm})=3.18\left(\mathrm{t} ; \mathrm{J}=7 \mathrm{~Hz}, 2 \mathrm{H},-\mathrm{CH}_{2}-\right)$, $3.87\left(\mathrm{~s} ; 3 \mathrm{H},-\mathrm{OCH}_{3}\right), 3.90\left(\mathrm{~s} ; 3 \mathrm{H},-\mathrm{OCH}_{3}\right), 3.97\left(\mathrm{~s} ; 3 \mathrm{H},-\mathrm{OCH}_{3}\right), 4.20(\mathrm{t}$; $\left.\mathrm{J}=7 \mathrm{~Hz}, 2 \mathrm{H},-\mathrm{CH}_{2}-\right), 6.70(\mathrm{~s} ; 1 \mathrm{H}$, vinyl-H), 6.83-7.02 (m; $3 \mathrm{H}, \mathrm{ArH})$, $7.12(\mathrm{~s} ; 1 \mathrm{H}, \mathrm{ArH}), 7.70(\mathrm{~d} ; \mathrm{J}=9 \mathrm{~Hz}, 1 \mathrm{H}, \mathrm{ArH})$.

\section{5,6-Dihydro-2,3,10-trimethoxy-indolo/2,1-alisoquinoline (7d)}

M.p. $217^{\circ}$. $-\mathrm{C}_{19} \mathrm{H}_{19} \mathrm{NO}_{3}(309.4)$ Calc. C $73.8 \mathrm{H} 6.19$ Found C 73.6 H 6.09. - ${ }^{1} \mathrm{H}-\mathrm{NMR}\left(\mathrm{CDCl}_{3}\right): \delta(\mathrm{ppm})=3.15\left(\mathrm{t} ; \mathrm{J}=7 \mathrm{~Hz}, 2 \mathrm{H},-\mathrm{CH}_{2}-\right)$, $3.90\left(\mathrm{~s} ; 3 \mathrm{H},-\mathrm{OCH}_{3}\right), 3.95\left(\mathrm{~s} ; 3 \mathrm{H},-\mathrm{OCH}_{3}\right), 4.00\left(\mathrm{~s} ; 3 \mathrm{H},-\mathrm{OCH}_{3}\right), 4.23(\mathrm{t}$; $\left.\mathrm{J}=7 \mathrm{~Hz}, 2 \mathrm{H},-\mathrm{CH}_{2}-\right), 6.73(\mathrm{~s} ; 1 \mathrm{H}$, vinyl-H), $6.83(\mathrm{~s} ; 1 \mathrm{H}, \mathrm{ArH}), 6.93-7.37$ (m; 4H, ArH).

\section{5,6-Dihydro-2,3,9-trimethoxy-indolo[2,1-a]isoquinoline (7e)}

M.p. $198^{\circ}$. $-\mathrm{C}_{19} \mathrm{H}_{19} \mathrm{NO}_{3}$ (309.4) Calc. C $73.8 \mathrm{H} 6.19$ Found $\mathrm{C} 73.5$ $\mathrm{H}$ 6.15. $-{ }^{1} \mathrm{H}-\mathrm{NMR}\left(\mathrm{CDCl}_{3}\right): \delta(\mathrm{ppm})=3.10\left(\mathrm{t} ; \mathrm{J}=7 \mathrm{~Hz}, 2 \mathrm{H},-\mathrm{CH}_{2}-\right)$, $3.88\left(\mathrm{~s} ; 3 \mathrm{H},-\mathrm{OCH}_{3}\right), 3.90\left(\mathrm{~s} ; 3 \mathrm{H},-\mathrm{OCH}_{3}\right), 3.95\left(\mathrm{~s} ; 3 \mathrm{H},-\mathrm{OCH}_{3}\right), 4.17(\mathrm{t}$; $\left.\mathrm{J}=7 \mathrm{~Hz}, 2 \mathrm{H},-\mathrm{CH}_{2}-\right), 6.73-6.83(\mathrm{~m} ; 4 \mathrm{H}, \operatorname{ArH}$, vinyl- $\mathrm{H}), 7.20(\mathrm{~s} ; 1 \mathrm{H}$, $\operatorname{ArH}), 7.52(\mathrm{~d} ; \mathrm{J}=9 \mathrm{~Hz}, 1 \mathrm{H}, \operatorname{ArH})$.

\section{5,6-Dihydro-2,3,9,10-tetramethoxy-indolo/2,1-a]isoquinoline (7f)}

M.p. $209-210^{\circ}$ (lit. 209-210 11$)$ ).

General Procedure for the Dehydrogenation of the Dihydroindoloisoquinolines to the Indolo/2,1-a/isoquinolines 8a-f with $\mathrm{Pd} / \mathrm{C}$

Dihydro-indolo[2,1-a]isoquinoline (1.3 mmol) and Pd/C $10 \%(150 \mathrm{mg})$ were mixed thoroughly in an agate mortar. This and all of the following operations were carried out under $\mathrm{N}_{2}$. A flask containing the mixture was placed in an oil bath of a temp. which was kept $10-15^{\circ}$ above the melting point of the dihydro-indoloisoquinoline. After $30 \mathrm{~min}$, the mixture was stirred with a spatula. Heating was continued for $30 \mathrm{~min}$. After cooling, the mixture was dissolved in $\mathrm{CH}_{2} \mathrm{Cl}_{2}$ and filtrated. The solvent was evaporated and the residue was chromatographed $\left(\mathrm{SiO}_{2} ; \mathrm{CH}_{2} \mathrm{Cl}_{2}\right)$. Recrystallization from EtOH afforded colorless crystals. The yields were $80-90 \%$.

\section{3,10-Dimethoxy-indolo/2,1-alisoquinoline (8a)}

Yield $80 \%$; m.p. 255-256.$-\mathrm{C}_{18} \mathrm{H}_{15} \mathrm{NO}_{2} \times 1 / 4 \mathrm{H}_{2} \mathrm{O}$ (281.4) Calc. C 76.8 H 5.55 Found C 76.8 H 5.53. - IR (KBr): $3420\left(\mathrm{H}_{2} \mathrm{O}\right) \mathrm{cm}^{-1} .{ }^{1} \mathrm{H}-$ $\mathrm{NMR}\left(\mathrm{CDCl}_{3}\right): \delta(\mathrm{ppm})=3.93\left(\mathrm{~s} ; 6 \mathrm{H},-\mathrm{OCH}_{3}\right), 6.62,7.67(\mathrm{AB} ; \mathrm{J}=9 \mathrm{~Hz}$, 2H, ArH), 6.87-7.24 (m;5H, ArH), 7.93-8.13 (m; 2H, ArH).

\section{3,9-Dimethoxy-indolo/2,1-a)isoquinoline (8b)}

Yield $85 \%$; m.p. $217^{\circ}$. $-\mathrm{C}_{18} \mathrm{H}_{15} \mathrm{NO}_{2} \times 1 / 4 \mathrm{H}_{2} \mathrm{O}$ (281.4) Calc. C 76.8 H 5.55 Found 76.7 H 5.69. $-{ }^{1} \mathrm{H}-\mathrm{NMR}\left(\mathrm{CDCl}_{3}\right): \delta(\mathrm{ppm})=3.90(\mathrm{~s} ; 3 \mathrm{H}$, $\left.-\mathrm{OCH}_{3}\right), 3.93\left(\mathrm{~s} ; 3 \mathrm{H},-\mathrm{OCH}_{3}\right), 6.5-7.23(\mathrm{~m} ; 6 \mathrm{H}, \mathrm{ArH}), 7.58-8.07(\mathrm{~m} ; 3 \mathrm{H}$, ArH).

\section{3,9,10-Trimethoxy-indolo/2,1-alisoquinoline (8c)}

Yield $80 \%$; m.p. $230^{\circ}$. $-\mathrm{C}_{19} \mathrm{H}_{17} \mathrm{NO}_{3} \times 1 / 4 \mathrm{H}_{2} \mathrm{O}$ (311.4) Calc. C 73.2 H 5.66 Found C 73.2 H 5.85. $-{ }^{1} \mathrm{H}-\mathrm{NMR}\left(\mathrm{CDCl}_{3}\right): \delta(\mathrm{ppm})=3.92(\mathrm{~s}$; $\left.3 \mathrm{H},-\mathrm{OCH}_{3}\right), 3.97\left(\mathrm{~s} ; 3 \mathrm{H}, \mathrm{OCH}_{3}\right), 4.01\left(\mathrm{~s} ; 3 \mathrm{H},-\mathrm{OCH}_{3}\right), 6.58,7.93(\mathrm{AB}$; $\mathrm{J}=9 \mathrm{~Hz}, 2 \mathrm{H}, \operatorname{ArH}), 6.93(\mathrm{~s} ; 1 \mathrm{H}, \operatorname{ArH}), 6.98(\mathrm{~s} ; 1 \mathrm{H}, \operatorname{ArH}), 7.15-7.26(\mathrm{~m}$; $3 \mathrm{H}, \mathrm{ArH}), 7.98$ (d; J $=9 \mathrm{~Hz}, 1 \mathrm{H}, \operatorname{ArH})$.

\section{2,3,10-Trimethoxy-indolo[ 2,1-alisoquinoline (8d)}

Yield $55 \%$; m.p. $217-218^{\circ}$. $-\mathrm{C}_{19} \mathrm{H}_{17} \mathrm{NO}_{3} \times 1 / 4 \mathrm{H}_{2} \mathrm{O}$ (311.4) Calc. C 73.2 H 5.66 Found C 73.3 H 5.75. - ${ }^{1} \mathrm{H}-\mathrm{NMR}\left(\mathrm{CDCl}_{3}\right): \delta(\mathrm{ppm})=$ $3.97\left(\mathrm{~s} ; 3 \mathrm{H},-\mathrm{OCH}_{3}\right), 4.05\left(\mathrm{~s} ; 3 \mathrm{H},-\mathrm{OCH}_{3}\right), 4.12\left(\mathrm{~s} ; 3 \mathrm{H},-\mathrm{OCH}_{3}\right), 6.72(\mathrm{~d} ; \mathrm{J}$ $=9 \mathrm{~Hz}, 1 \mathrm{H}, \mathrm{ArH}), 6.97-7.37(\mathrm{~m} ; 4 \mathrm{H}, \mathrm{ArH}), 7.60(\mathrm{~s} ; 1 \mathrm{H}, \mathrm{ArH}), 7.80(\mathrm{~d} ; \mathrm{J}$ $=9 \mathrm{~Hz}, 1 \mathrm{H}, \operatorname{ArH}), 8.10(\mathrm{~d} ; \mathrm{J}=9 \mathrm{~Hz}, 1 \mathrm{H}, \operatorname{ArH})$.

\section{2,3,9-Trimethoxy-indolo/2,1-alisoquinoline (8e)}

Yield $60 \%$; m.p. $217^{\circ}$. $-\mathrm{C}_{19} \mathrm{H}_{17} \mathrm{NO}_{3} \times 1 / 4 \mathrm{H}_{2} \mathrm{O}(311.4)$ Calc. $\mathrm{C} 73.2$ H 5.66 Found C $73.0 \mathrm{H}$ 5.66. $-{ }^{1} \mathrm{H}-\mathrm{NMR}\left(\mathrm{CDCl}_{3}\right): \delta(\mathrm{ppm})=3.98(\mathrm{~s}$; $\left.3 \mathrm{H},-\mathrm{OCH}_{3}\right), 4.02\left(\mathrm{~s} ; 3 \mathrm{H},-\mathrm{OCH}_{3}\right), 4.08\left(\mathrm{~s} ; 3 \mathrm{H},-\mathrm{OCH}_{3}\right), 6.63,7.93(\mathrm{AB} ; \mathrm{J}$ $=9 \mathrm{~Hz}, 2 \mathrm{H}, \mathrm{ArH}), 6.97-7.30(\mathrm{~m} ; 4 \mathrm{H}, \mathrm{ArH}), 7.53(\mathrm{~s} ; 1 \mathrm{H}, \operatorname{ArH}), 7.73(\mathrm{~d} ; \mathrm{J}$ $=9 \mathrm{~Hz}, 1 \mathrm{H}, \mathrm{ArH})$.

\section{2,3,9,10-Tetramethoxy-indolo/ 2,1-a)isoquinoline (8f)}

Yield $75 \%$; m.p. $210^{\circ}$. $-\mathrm{C}_{20} \mathrm{H}_{19} \mathrm{NO}_{4} \times 1 / 4 \mathrm{H}_{2} \mathrm{O}$ (341.4) Calc. $\mathrm{C} 70.3$ H 5.62 Found C $70.3 \mathrm{H} \mathrm{5.68.}-{ }^{1} \mathrm{H}-\mathrm{NMR}\left(\mathrm{CDCl}_{3}\right): \delta(\mathrm{ppm})=3.98(\mathrm{~s}$; $\left.3 \mathrm{H},-\mathrm{OCH}_{3}\right), 4.02\left(\mathrm{~s} ; 6 \mathrm{H},-\mathrm{OCH}_{3}\right), 4.07\left(\mathrm{~s} ; 3 \mathrm{H},-\mathrm{OCH}_{3}\right), 6.63,7.95(\mathrm{AB}$; $\mathrm{J}=9 \mathrm{~Hz}, 2 \mathrm{H}, \operatorname{ArH}), 6.92(\mathrm{~s} ; 1 \mathrm{H}, \operatorname{ArH}), 7.0(\mathrm{~s} ; 1 \mathrm{H}, \operatorname{ArH}), 7.20(\mathrm{~s} ; 1 \mathrm{H}$, ArH), 7.28 (s; 1H, ArH), 7.48 (s; 1H, ArH).

\section{Biological methods}

P388 D Leukemia Cells ${ }^{12)}$

Murine P388 $D_{1}$ leukemia cells were cultured in RMPI 1640 medium (Biochrom, Berlin) supplemented with 10 mM HEPES* buffer, $10 \%$ desactivated horse serum (Biochrom), $2 \mathrm{mM}$ glutamine and $0.085 \%$ $\mathrm{NaHCO}_{3}$. Cells were grown in an incubator in $5 \% \mathrm{CO}_{2}$ at $37^{\circ}$. Aliquots of $2 \mathrm{ml}$ of the cell suspension containing 7-8 $\times 10^{4}$ cells were plated in test tubes. Substances dissolved in $2 \mu \mathrm{l}$ of DMSO were added. The medium of control wells contained an equal volume of DMSO. After two days of incubation, cells were labeled for $2 \mathrm{~h}$ with $0.3 \mu \mathrm{Ci}{ }^{3} \mathrm{H}$-thymidine (NEN) per well. $1 \mathrm{ml}$ was used for determination of cell number (Coulter counter $\mathrm{ZM}$ ). From the remaining part cells were harvested by centrifugation, washed with PBS and sonicated (Branson). After addition of $4 \mathrm{ml}$ of $10 \%$ trichloroacetic acid, the acid-insoluble fraction was collected on a $0.4 \mu \mathrm{m}$ filter (Sartorius) and counted after addition of $10 \mathrm{ml}$ scintillation liquid (Quickszint 212, Zinnser) in a LS 1801 scintillation counter (Beckman).

* HEPES: 4-(2-Hydroxyethyl)-1-piperazineethanesulfonic acid

\section{MDA-MB 231 Human Breast Cancer Cells ${ }^{13)}$}

Cells were grown in $\mathrm{McCoy} 5$ a medium (Boehringer, Mannheim) supplemented with $10 \%$ newborn calf serum (NCS) (Gibco) and gentamycine $(40 \mu \mathrm{g} / \mathrm{ml})$. Cells were grown in a humidified incubator in $5 \% \mathrm{CO}_{2}$ at $37^{\circ}$. Cells were harvested with $0.05 \%$ trypsine- $0.02 \%$ EDTA in $0.15 \mathrm{M} \mathrm{NaCl}$ and approximately $2 \times 10^{4}$ cells in $2 \mathrm{ml}$ were plated in six-well dishes (Linbro). Two days later cells were switched to a medium containing $5 \% \mathrm{NCS}$ and the substances, dissolved in $2 \mu \mathrm{lDMSO}$. The medium of control wells contained an equal volume of DMSO. Two days later, cells were labeled for $2 \mathrm{~h}$ with $0.3 \mu \mathrm{Ci}{ }^{3} \mathrm{H}$-thymidine per well. $1 \mathrm{ml}$ was used for determination of cell number. The remaining cells were harvested by centrifugation, washed with PBS and sonicated. After addition of $4 \mathrm{ml}$ of $10 \%$ trichloroacetic acid, the acid insoluble fraction was collected on a $0.4 \mu \mathrm{m}$ filter and counted after addition of $10 \mathrm{ml}$ scintillation liquid in a scintillation counter. 


\section{References}

1 J. B. LePecq, Nguyen-Dat-Xuong, C. Gosse, and C. Paoletti, Proc. Nat. Acad. Sci. USA 71, 5078 (1974).

2 E. von Angerer and J. Prekajac, J. Med. Chem. 29, 380 (1986).

3 P. Chinnasamy, K. Iwasa, S. von Angerer, C. Weimar, and W. Wiegrebe, Arch. Pharm. (Weinheim) 320, 790 (1987).

4 J. S. Buck, R. D. Haworth, and W. H. Perkin jun., J. Chem. Soc. (London) 125, 2176 (1924).

5 S. Kano, E. Komiyama, K. Nawa, and S. Shibuya, Chem. Pharm. Bull. 24, 310 (1976).
6 A. Pschorr, Liebigs Ann. Chem. 391, 51 (1912).

7 R. G. Naik and T. S. Wheeler, J. Chem. Soc. 1938, 1780.

8 H. Kondo and Uyeo, J. Pharm. Soc. Japan 53, 557 (1933).

9 L. Helfer, Helv. Chim. Act. 7, 945 (1924).

10 T. Kametani and M. Ihara, J. Chem. Soc. C, 530 (1967).

1 I C. P. Mak and A. Brossi, Heterocycles 12, 1413 (1979).

12 W. Meindl, R. Laske, and M. Böhm, Arch. Pharm. (Weinheim) 320, 730 (1987).

13 E. von Angerer, J. Prekajac, and M. Berger, Eur. J. Canc. Clin. Onc. 21,531 (1985).

[Ph 446] 